\title{
Relationship between Sleep and Subjective Fatigue in Rotating Shift Nurses: Validation Using a Wearable Device
}

\author{
Yuko Yoshida $^{*}$, Naomi Sumi ${ }^{1}$, Naotaka Sugimura ${ }^{2}$, Fumie Nemoto ${ }^{3}$, Rika Yano ${ }^{1}$ \\ ${ }^{1}$ Faculty of Health Sciences, Hokkaido University, Sapporo, Japan \\ ${ }^{2}$ Graduate School of Health Sciences, Hokkaido University, Sapporo, Japan \\ ${ }^{3}$ Tokeidai Memorial Hospital, Sapporo, Japan \\ Email: *yuko790402@hs.hokudai.ac.jp
}

How to cite this paper: Yoshida, Y., Sumi, N., Sugimura, N., Nemoto, F. and Yano, R. (2018) Relationship between Sleep and Subjective Fatigue in Rotating Shift Nurses: Validation Using a Wearable Device. Open Journal of Nursing, 8, 272-280.

https://doi.org/10.4236/ojn.2018.84023

Received: March 162018

Accepted: April 27, 2018

Published: April 30, 2018

Copyright $\odot 2018$ by authors and Scientific Research Publishing Inc. This work is licensed under the Creative Commons Attribution International License (CC BY 4.0).

http://creativecommons.org/licenses/by/4.0/

\begin{abstract}
The objective of this study was to determine if sleep indicators are associated with subjective fatigue in nurses working in a rotating, two-shift system with a 16-hour night shift, using the wearable device Fitbit One. Subjective fatigue was evaluated using the Subjective Fatigue Symptom Questionnaire (SFSQ), a rating scale developed by the Working Group for Occupational Fatigue of the Japan Society for Occupational Health. Subjects were asked to continuously wear a Fitbit One to obtain the following data: time in bed (TIB), deep sleep time, length and frequency of nighttime awakening, sleep efficiency, waking time, and bed time. Nurses had a mean age and standard deviation of $33.2 \pm 7.5$ years and had worked as nurses for $9.8 \pm 6.2$ years on average. Eight nurses were in their 20s (42.1\%), 5 in their 30 s (26.3\%), and 6 in their 40 s (31.6\%). All participants were female. Participants' SFSQ scores calculated from the 9 evenings in the study period were averaged to establish a threshold for categorizing high-fatigue (HF) and low-fatigue (LF) groups. No significant differences were found between HF and LF nurses in terms of TIB, deep sleep time, nighttime awakening time or frequency, or sleep efficiency on any type of day (as classified by shift, day off, etc.). Nurses in both groups woke up significantly later on days off and on pre-night-shift days than on day-shift days. These results demonstrate the variation in sleep patterns of nurses on a rotating, two-shift system between day-shift and night-shift days.
\end{abstract}

\section{Keywords}

Fatigue, Rotating Shifts, Nurse, Sleep 


\section{Introduction}

Excessive fatigue not only causes nurses to develop health problems, but it also negatively impacts the safety of medical care by reducing their work efficiency and making them more prone to making medical errors. Nurses who work in a rotating shift system may be required to work at night when they would normally be asleep. Night shifts place nurses under mental and physical stress that exceed those experienced when working during the day. Daytime sleep does not improve fatigue as much as nighttime sleep [1].

In Japan, an increasing proportion of hospital wards operate under a rotating two-shift system, characterized by a long night-shift (of greater than 8 hours). Initially, the system was introduced to chronic wards that focused mostly on long-term medical care, but this trend has spread to acute and advanced wards. At present, more than half of rotating two-shift systems incorporate a night shift of 16 hours or more [2].

Wearable devices have attracted attention in recent years as potential tools for health management. Studies have demonstrated the validity of many of these devices and clinical applications are anticipated. One such wearable device is the Fitbit One (Fitbit; San Francisco, CA, USA). This is an extremely compact device that weighs only $13.4 \mathrm{~g}$ and measures $48 \times 19.3 \mathrm{~mm}$ in size. Wearing this device is minimally stressful, as it can be attached to the underwear or other clothing. In addition to activity levels, this device can measure various sleep-related metrics such as overall sleep time, nighttime awakening time and frequency, and sleep efficiency. These data can be displayed using a smartphone or computer, allowing users to self-monitor and track their behavior in an intuitive manner. The Fitbit One has previously been applied to measure activity levels in patients with heart disease, as well as in patients undergoing rehabilitation [3] [4].

In the present study, we investigated the associations between subjective fatigue and sleep indicators measured using this wearable device in rotating shift nurses, in an effort for these findings to serve as a basic reference for a potential simple method to identify early warning signs of fatigue in nurses.

\section{Aim}

To investigate the association between sleep indicators and subjective fatigue in nurses working in a rotating two-shift system with a 16-hour night-shift using the Fitbit One.

\section{Methods}

\subsection{Subjects}

Subjects were recruited from an acute-care hospital that uses a rotating two-shift system characterized by a 16-hour night-shift. Specifically, the day-shift lasts from 0900 to 1700 hours, and the night-shift lasts from 1700 to 0900 hours on the following morning. Chief nurses of the hospital's internal medicine and surgical wards were asked to recommend nurses not having extended vacation dur- 
ing the study period as candidates. Any nurses who were taking sleep medication or receiving treatment for any disease were excluded from this study. Official requests for research cooperation were sent to the list of candidates via email and by paper, and nurses were recruited upon consenting to study participation.

\subsection{Survey Items}

\section{1) Basic Characteristics}

Subjects were asked their age, sex, height, weight, years of nursing experience, whether they were living with anyone, and whether they were taking any medications other than for sleep when they were fitted with the Fitbit One.

2) Sleep Indicators (Fitbit One)

Participating nurses were asked to keep the wearable device Fitbit One affixed to their clothes for 9 consecutive days, during both working and non-working hours. Data recorded by Fitbit One included calorie expenditure, time in bed (TIB), deep sleep time, and nighttime awakening time and frequency; sleep efficiency was additionally calculated based on this data (i.e., deep sleep time/TIB $\times$ 100). Below, days on which nurses began a night shift are called "pre-night shift" days, while the days on which they ended a 16-hour night shift are called "post-night shift" days. Wake-up time and bed time were also recorded, which are factors that can contribute to fatigue [5]. Overall sleep time was calculated from the Fitbit One data. For example, consider a nurse who slept at 2200 on Day 0, and woke up at 0600 on Day 1. This would be calculated not as Day $0=2$ $\mathrm{h}$ and Day $1=6 \mathrm{~h}$, but simply as Day $1=8 \mathrm{~h}$. Sleep time in this study means the subject's longest sleep time each day; this indicator is automatically recorded by the Fitbit app as "main sleep".

3) Subjective Fatigue (SFSQ)

Subjective fatigue was evaluated using the Subjective Fatigue Symptom Questionnaire (SFSQ), a rating scale developed by the Working Group for Occupational Fatigue of the Japan Society for Occupational Health [6]. The SFSQ contains 25 question items corresponding to specific complaints observed in subjective fatigue, grouped into 5 categories: drowsiness, instability, uneasiness, local pain or dullness, and eye strain. Respondents rate each of the symptoms according to how strongly they perceive them on a 5-point scale characterized by: "Strongly disagree" ( 1 point) to "Strongly agree" ( 5 points). Higher category and overall scores indicate greater fatigue [6]. Nurses were asked to fill out the SFSQ before starting and after completing their shift on work days, and upon waking up and before going to bed on days off. To establish a basis for analysis, all participants' total SFSQ scores completed in the evening were averaged: nurses above the median average were assigned to a high fatigue (HF) group, while nurses below the median were assigned to a low fatigue (LF) group.

\subsection{Study Period}

To the study was conducted between November 2015 and May 2016. 


\subsection{Analysis Method}

All statistical analyses, including descriptive statistics, Mann-Whitney U test and Kruskal-Wallis tests, were conducted using SPSS statistical software (version. 22; IBM Corp., Armonk, NY, USA).

\subsection{Ethical Considerations}

Surveys and Fitbit One data were anonymized and managed using ID numbers. A reference sheet corresponding to subject names and ID numbers was stored in a locked document storeroom. Fitbit One devices were worn for the duration of the study as described in the Methods, but participants were told they could remove them as desired when they felt constrained or encumbered. Subjects were asked to provide researchers with a paper record of the time(s) spent not wearing the device under this scenario. Nurses were explained verbally and in writing that the data obtained would not be used for any other purpose besides this study and all data would be deleted at the conclusion of the study. Written consent was obtained from all participants. This research was conducted with the approval of the ethics committees of the Hokkaido University Graduate School of Health Sciences, Faculty of Health Sciences and the participating hospital.

\section{Results}

\subsection{Subject Characteristics (Table 1)}

Nurses had a mean age of $33.2 \pm 7.5$ years and had worked as nurses for $9.8 \pm 6.2$ years on average. Eight were in their 20's (42.1\%), 5 in their 30's (26.3\%), and 6 in their 40's (31.6\%). All participants were female. The average subject body-mass index (BMI) was $21.5 \pm 3.0$. No participants were caring for a child or living with a relative requiring long-term care. Four participants were taking medications for reasons other than sleep (21.1\%). Three nurses had only 1 night-shift during the 9-day study period (15.8\%), 12 had 2 night-shifts (63.2\%), and 4 had 3 night-shifts (21.1\%). Three nurses had only 1 day off during the study period (15.8\%), 10 had 2 nights off (63.2\%), 3 had 3 nights off (15.8\%), and 3 had 4 nights off (15.8\%). The LF and HF groups had 9 and 10 members, respectively. No significant differences between the two groups were observed for any basic subject characteristic.

\subsection{Subjective Fatigue Symptoms (SFSQ)}

The median mean score for SFSQs taken in the evenings was 48.9 points; nurses scoring higher and lower were respectively analyzed as members of the HF and LF groups, respectively. Scores and sub-scores were compared using the Mann-Whitney U test. Significant differences were not only observed in terms of total SFSQ score, but also in each of the sub-scores (drowsiness, instability, uneasiness, local pain or dullness, eye strain). 
Table 1. Participants' basic characteristics.

\begin{tabular}{|c|c|c|c|c|}
\hline & $\begin{array}{l}\text { All participants } \\
\qquad(\mathrm{n}=19)\end{array}$ & $\begin{array}{l}\text { Low fatigue } \\
\qquad(\mathrm{n}=9)\end{array}$ & $\begin{array}{l}\text { High fatigue } \\
\quad(\mathrm{n}=10)\end{array}$ & p-values \\
\hline Age (y) & $33.2 \pm 7.5$ & $32.4 \pm 7.7$ & $33.8 \pm 7.7$ & 0.62 \\
\hline $20-29$ & $8(42.1)$ & $5(55.6)$ & $3(30.0)$ & \multirow[t]{3}{*}{0.52} \\
\hline $30-39$ & $5(26.3)$ & $2(22.2)$ & $3(30.0)$ & \\
\hline $40-49$ & $6(31.6)$ & $2(22.2)$ & $4(40.0)$ & \\
\hline Nursing experience (y) & $9.8 \pm 6.2$ & $11.07 \pm 7.7$ & $4.0 \pm 2.8$ & 0.74 \\
\hline \multicolumn{5}{|l|}{$\begin{array}{c}\text { Medications } \\
\text { (other than for sleeping) }\end{array}$} \\
\hline Yes & $4(21.1)$ & $3(30.3)$ & $1(10.0)$ & \multirow{2}{*}{0.30} \\
\hline No & $15(78.9)$ & $6(66.7)$ & $9(90.0)$ & \\
\hline BMI & $21.5 \pm 3.0$ & $21.7 \pm 2.9$ & $21.3 \pm 3.3$ & 0.65 \\
\hline \multicolumn{5}{|l|}{$\begin{array}{l}\text { Night shifts during study } \\
\text { period }\end{array}$} \\
\hline 1 & $3(15.8)$ & $2(22.2)$ & $1(10.0)$ & \multirow{3}{*}{0.71} \\
\hline 2 & $12(63.2)$ & $5(55.6)$ & $7(70.0)$ & \\
\hline 3 & $4(21.1)$ & $2(22.2)$ & $2(20.0)$ & \\
\hline \multicolumn{5}{|l|}{$\begin{array}{l}\text { Days off during study } \\
\text { period }\end{array}$} \\
\hline 1 & $3(15.8)$ & $2(22.2)$ & $1(10.0)$ & \multirow{4}{*}{0.47} \\
\hline 2 & $10(52.6)$ & $3(33.3)$ & $7(70.0)$ & \\
\hline 3 & $3(15.8)$ & $2(22.2)$ & $1(10.0)$ & \\
\hline 4 & $3(15.8)$ & $2(22.2)$ & $1(10.0)$ & \\
\hline
\end{tabular}

Categorical data: $\mathrm{n}(\%)$; continuous data: mean $\pm \mathrm{SD}$, Age, nursing experience, BMI: Mann-Whitney U tests. Age group, number of night shifts, days off: Chi-square tests. Medications (other than for sleeping): Fisher's exact test.

\subsection{Sleep Patterns by Type of Working Day}

No significant differences were found between nurses in the HF and LF groups in terms of TIB, deep sleep time, nighttime awakening time or frequency, or sleep efficiency on days of the same type. Sleep efficiency ranged from $94 \%$ $96 \%$ in both groups. Nurses in both groups slept deeply for less time on post-night-shift days than on other work days and days off (Figure 1). LF nurses had low variations in deep sleep time on the days when they worked a daytime shift. In both groups, nighttime awakening time was significantly shorter on post-night-shift days than on pre-night-shift days or days off (Figure 2). Nurses likely had shorter arousal times on post-night-shift days as a consequence of sleeping for less time overall (Figure 1, Figure 2).

The wake-up and bed times are shown in Table 2. Significant differences were not observed in terms of wake-up time between the HF and LF groups, when compared on the same type of days. Nurses in both groups woke up significantly later on days off and on pre-night-shift days than on days working the day-shift.

Figure Labels: Use 8 point Times New Roman for Figure labels. Use words rather than symbols or abbreviations when writing Figure axis labels to avoid. 
Table 2. Wake-up time and bed time by type of working day.

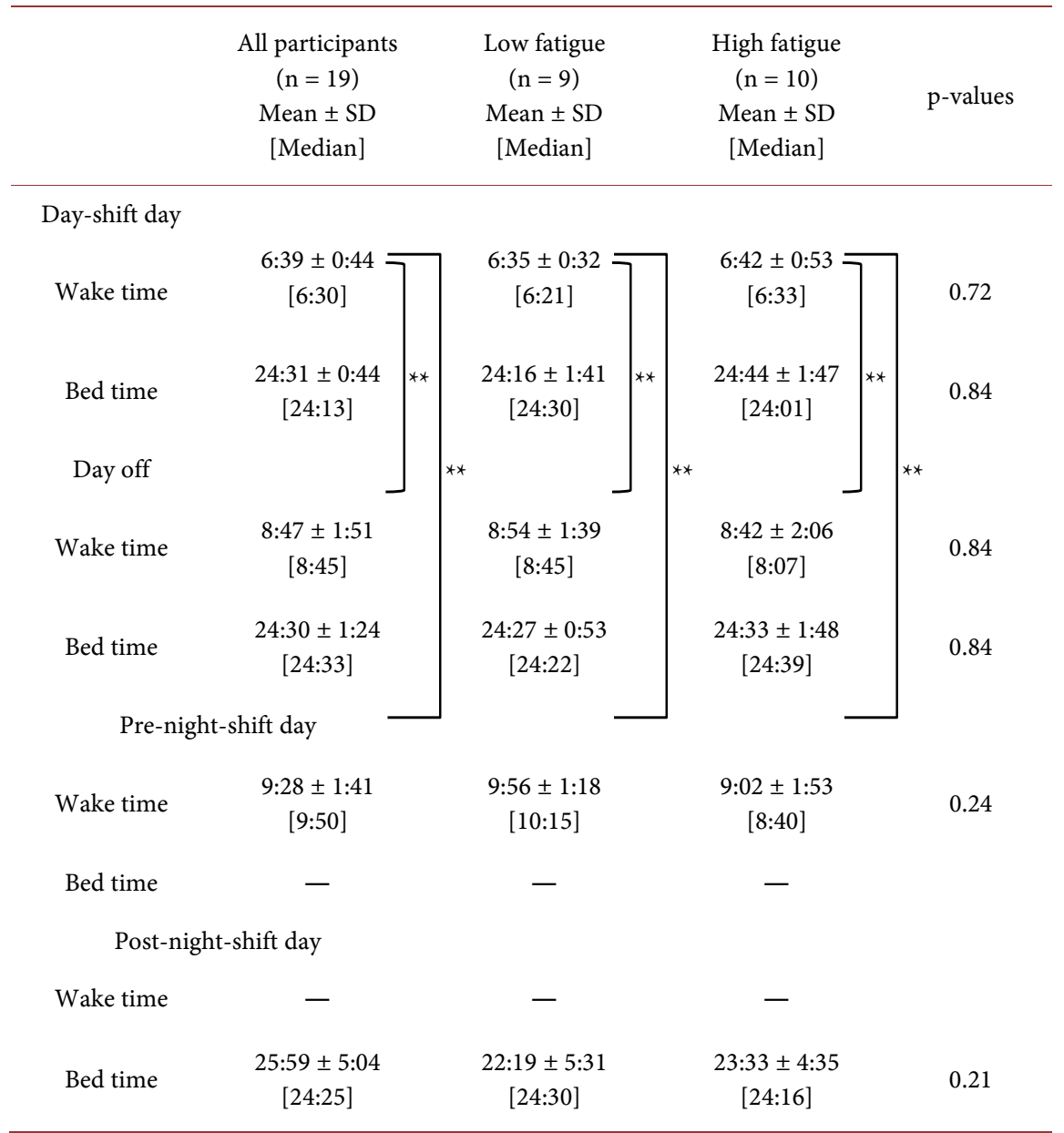

${ }^{* *} \mathrm{p}<0.01$, Kruskal-Wallis test was used to compare the type of working days, Mann-Whitney U test used to compare differences between the HF and LF groups.

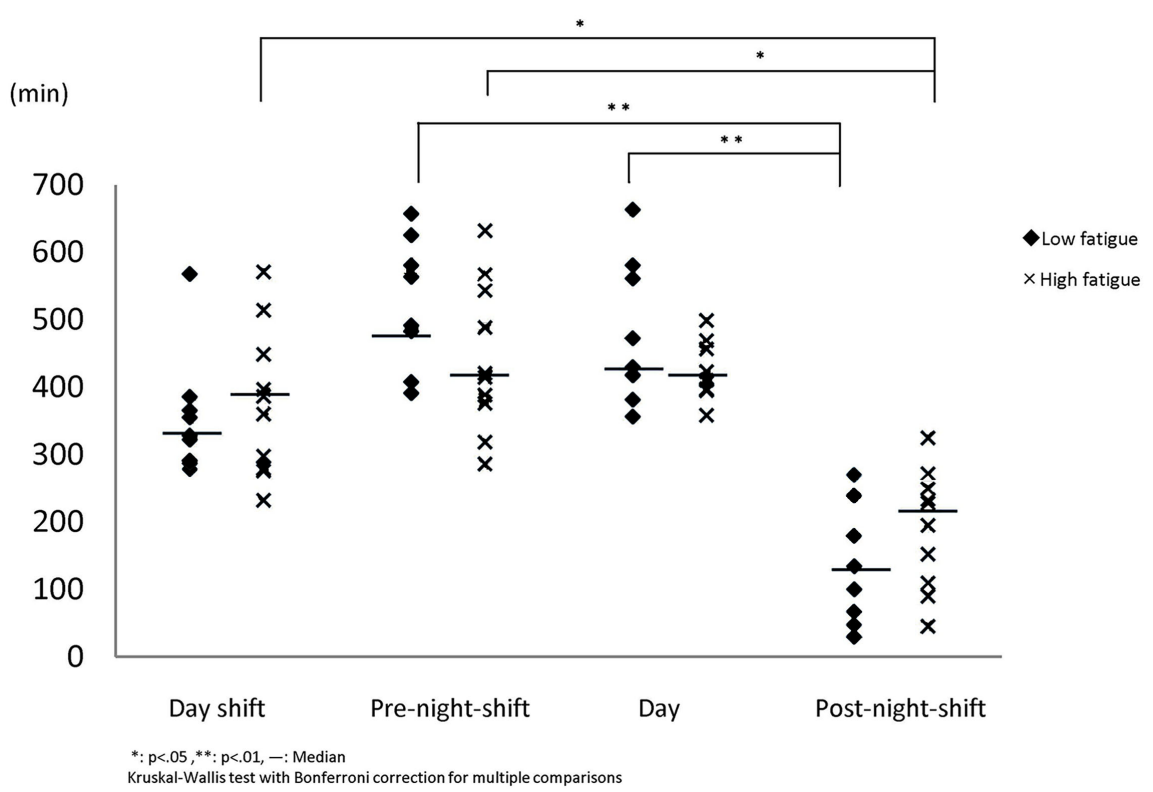

Figure 1. Deep sleep time: day type comparisons of low fatigue and high fatigue. 


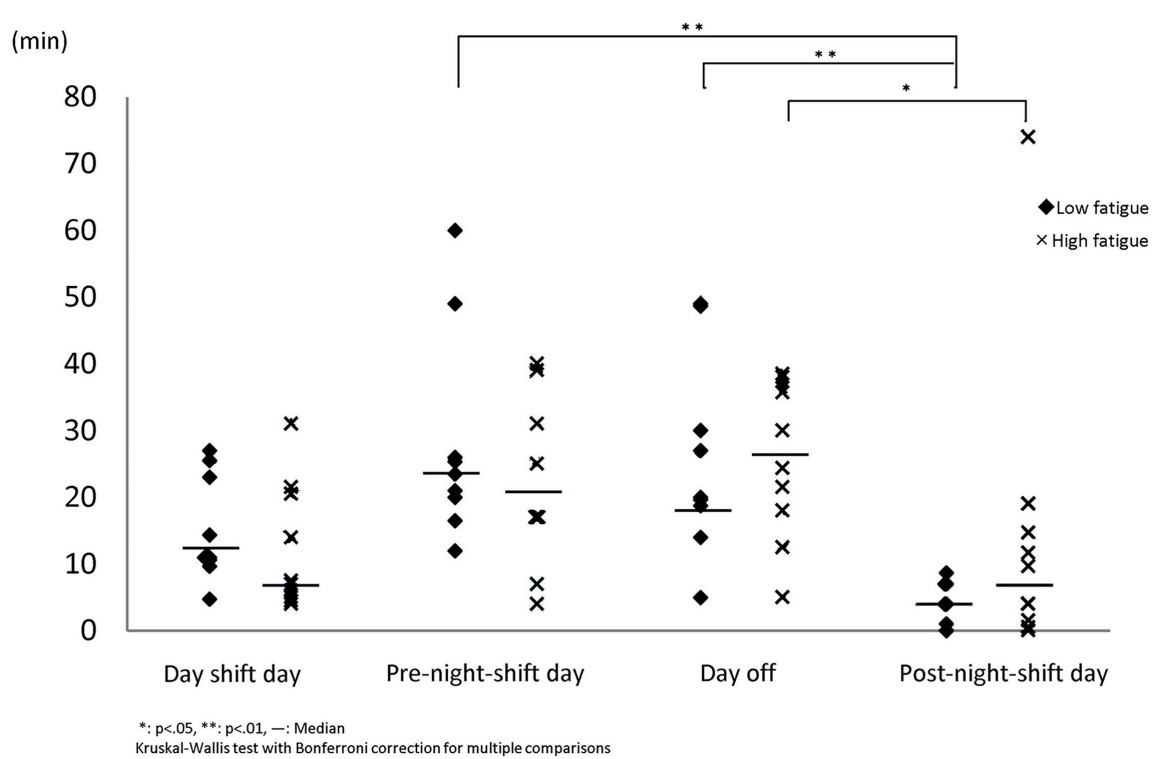

Figure 2. Nighttime awakening time: day type comparisons about low fatigue and high fatigue.

\section{Discussion}

\subsection{Subjective Fatigue and Sleep Indicators}

No significant difference in any sleep indicator was observed between nurses in the HF versus LF group in the present study. The data did reveal one trend, i.e., nurses woke up later on days off and pre-night-shift days than on day-shift days, but no findings clearly differentiated highly fatigued versus less-fatigued individuals in this regard. Wake-up time was related to the type of shift worked on the day in question; however, bed time on a given day was likely more affected by the type of shift on the next day than the shift-type on the day of data collection.

According to the 2015 National Health and Nutrition Survey, a majority of both Japanese men and women reported sleeping between "6 to 7 hours" a night on average [7]. This report's "sleep time" corresponds to TIB and deep sleep time in the present study, and our subjects' deep sleep time on days working the day-shift largely agrees with published government statistics (Figure 1). However, nurses sleep deeply for longer durations on pre-night-shift days and days off, on average, compared to the values reported by the Ministry of Health. Table 2 shows that subjects woke up approximately 2 hours later on days off than on days working the day-shift. Later wake-up times resulted in longer in-bed times and deep sleep times (i.e. sleep time) on days off than on days working the day-shift. Since rotating shift workers do not work the same shift every day, their daily rhythms are not constant, creating variability in sleep patterns between days they work the day-shift versus days when they work the night-shift. Therefore, the data presented here should not be directly compared with data for workers in general, who tend to work during the day and sleep at night. 


\subsection{Fitbit One Data Collection}

This study revealed the sleep patterns of nurses working in a rotating two-shift system to be characterized by either short overall sleep time, or heavily interrupted sleep, on post-night-shift days. One of the indicators tracked by Fitbit devices is "main sleep," which is calculated as the longest time the wearer spends continuously asleep on a given day (In May 2016, the calculation method for sleep time was modified to sum all sleep episodes, even those of short durations). As a result, nurses' sleep time (i.e., Fitbit's "main sleep") on post-night-shift days corresponded to a nap they took during work in some cases, but also as a nap taken after leaving the hospital in others. In addition, in the event that the wearer took multiple short naps, the device would only present data for the longest of these episodes for that day. With these limitations in mind, researchers should take caution when collecting sleep data on the day after a night-shift measured using the Fitbit One, noting that the sleep time shown could be less than the actual sleep time.

Nurses were found to have shorter nighttime awakenings in this study than noted in previous studies. When going to bed, Fitbit One users place the device inside the pocket of a special wristband they attach to their non-dominant hand. Using acceleration sensors, the Fitbit One records as "sleep interruptions" those times when the wearer frequently rolls over and moves around in bed. Therefore, researchers should be aware that this device is actually measuring the degree of "tossing and turning" at night, not arousal per se.

\section{Conclusions}

1) There was no relationship between subjective fatigue and sleep indicators in nurses classified by either HF or LF.

2) The sleeping patterns of nurses working a rotating two-shift system were found to be characterized by a) either short sleep time or heavily interrupted sleep on post-night shift days; and b) waking up later on days off or night-shift days than on days working the day-shift.

3) There are methodological difficulties inherent in interpreting Fitbit One sleep data on days when rotating shift nurses finish their night shifts; however, wake-up time and bed time were accurately tracked in the present study.

\section{Conflicts of Interest}

The authors have no conflicts of interest to declare.

\section{Research Funding}

This study was supported by MIC/SCOPE \#152301001.

\section{References}

[1] Matsumoto, S., Sasaki, T., Sakita, M., Naito, K., Aoyagi, N., Takahashi, E. and Sakai, K. (2008) The Effects of Naps Taken by Hospital Nurses During 16-Hour Night- 
shifts on Their Subjective Fatigue Feelings and Subsequent Sleeps. Journal of Science of Labour, 84, 25-29. (In Japanese)

[2] Japan Federation of Medical Worker's Unions (2014) Iryo Rodo. http://irouren.or.jp/publication/iryorodo584.pdf

[3] Takacs, J., Pollock, C.L., Guenther, J.R., Bahar, M., Napier, C. and Hunt, M.A. (2014) Validation of the Fitbit One activity Monitor Device during Treadmill Walking. Journal of Science and Medicine in Sport, 17, 496-500.

https://doi.org/10.1016/j.jsams.2013.10.241

[4] Cadmus-Bertram, L.A., Marcus, B.H., Patterson, R.E., Parker, B.A. and Morey, B.L. (2015) Randomized Trial of a Fitbit-Based Physical Activity Intervention for Women. American Journal of Preventive Medicine, 49, 414-418. https://doi.org/10.1016/j.amepre.2015.01.020

[5] Miyazaki, M. and Hosona, M. (2013) The Relationship between Sleeping Patterns and Fatigue among Nurses on Two-Shift Work Schedules. Bulletin of Health Sciences Kobe, 29, 1-15. (In Japanese)

[6] Kubo, T., Tachi, N., Takeyama, H., Ebara, T., Inoue, T., Takanishi, T., Arakomo, Y., Murasaki, G. and Itani, T. (2008) Characteristic Patterns of Fatigue Feelings on Four Simulated Consecutive Night Shifts by “Jikaku-sho Shirabe”. Sangyo Eiseigaku Zasshi, 50, 133-144. (In Japanese)

[7] Japan Ministry of Health, Labour and Welfare (2015) Summary of the 2015 National Health and Nutrition Survey Results.

http://www.mhlw.go.jp/bunya/kenkou/eiyou/h27-houkoku.html 